\title{
Twenty-First Century In Vitro Toxicology Testing Methods and the Assessment of e-Cigarettes
}

\author{
Moderator: John "Jack" R. Fowle, III \\ Participants: Rodger D. Curren, Thomas Hartung, ${ }^{3}$ Christopher Proctor, ${ }^{4}$ and Neil Wilcox ${ }^{5}$
}

\section{Introduction}

A N EXPERT ROUNDTABLE DISCUSSION was convened by Applied In Vitro Toxicology to address the increasingly important topic of the application of in vitro and other alternative toxicology approaches to help the U.S. Food and Drug Administration (FDA) and others evaluate the potential risks of next-generation nicotine products.

Dr. Fowle: Thanks all for joining the panel on this timely subject. Thomas, please could you lead off responses to the first question: How can 21st century in vitro toxicology efforts offer approaches and challenges for the assessment of e-cigarettes?

Dr. Hartung: First of all, e-cigarettes are an excellent example that the 21 st century brings new products and new needs for their risk assessment and regulation.

The first thing I think we need to agree on is that 20th century toxicity evaluation approaches are not suitable for these types of challenges. They are not only unsuitable because of the acceptance of animal models is decreasing with consumers, they are also not acceptable because we increasingly understand the shortcomings of these predictions with respect to humans and with respect to the costs and duration of the traditional animal-based approaches.

We know that we are facing something around 10,000 different flavors of e-cigarettes, and it is not possible to use these 20th century methodologies to do the respective assessments, and we cannot wait the 4 or 5 years for cancer bioassays to tell us something about these types of products.

The 21st century approach is based on incorporating the technologies of the 21st century and an increased understanding of mechanisms of toxicity. There are opportunities for embracing technologies developed for industrial chemicals, increasingly embraced for other things such as pesticides and drugs, which bring high-content technologies-technologies, which deliver content so that we can actually understand what the biological profile of this substance is, and higher throughput, which allows us to test at least hundreds of these substances in a reasonable timeframe.

And for this reason, this is an excellent engine for $21 \mathrm{st}$ century toxicology to meet an important area of consumer product use.

Dr. Proctor: I agree with Thomas that these products, in particular, give us a really interesting and important opportunity. With electronic cigarettes, the key issue is that, as Thomas said, there is a large variety of products currently on the market that, particularly when they are made by small companies, are unlikely to have been subject to very much safety testing to date. Further there is a large range of e-liquids, that are used with all sorts of different devices, and the devices are rapidly evolving.

We have seen, even in the past 5 years, an enormous range of different types of devices coming to market. So unless we can find some ways in which you can give fast throughput screening from a toxicological point of view, you really are going to be fairly blind to these things.

The other key thing to mention is that e-cigarettes are unlikely to be completely risk free, and there may be some devices and liquids already on the market that are riskier than others. It is just that we do not have a regulatory agreed set of tests yet to really be able to identify them and potentially screen them out.

Dr. Wilcox: I would endorse what has been said so far. Thomas described what we would call the macro picture. When you submit a product for approval, at least in the United States, there is going to be more of a microassessment and, that is, specifically: What are the characteristics of the device and what is being delivered? That is where the rubber hits the road.

Dr. Curren: I would elaborate on one point that Thomas so nicely made, which was the addition of tissues and human models into toxicity testing-with the use of human

\footnotetext{
${ }^{1}$ Science to Inform, LLC, Pittsboro, North Carolina.

${ }^{2}$ Institute for In Vitro Sciences, Inc., Gaithersburg, Maryland.

${ }^{3}$ Evidence-Based Toxicology, Doerenkamp-Zbinden, Department of Environmental Health and Engineering, Center for Alternatives to Animal Testing, Johns Hopkins Bloomberg School of Public Health, Baltimore, Maryland.

${ }_{5}^{4}$ Scientific Product Stewardship, British American Tobacco, Southampton, United Kingdom.

${ }^{5}$ Low Country Consulting, Bluffton, South Carolina.

(C) John R. Fowle, III et al., 2017; Published by Mary Ann Liebert, Inc. This Open Access article is distributed under the terms of the Creative Commons Attribution Noncommercial License (http://creativecommons.org/licenses/by-nc/4.0/), which permits any noncommercial use, distribution, and reproduction in any medium, provided the original author and the source are credited.
} 
in vitro models, at various levels of complexity, we conceivably would be able to align toxicity markers that we see in the in vitro model directly with similar end points that are seen in clinical tests with these devices.

This is an important additive activity that is really impossible when using the animal model.

Dr. Fowle: Excellent responses, all. The next question is: How is regulation globally impacting e-cigarette in vitro research?

Dr. Proctor: Let me start first with the variety of regulations that have been introduced over the past few years. They range from complete prohibition, for fear that e-cigarettes may cause adverse effects on the population, to fairly permissive regulations, particularly in the United Kingdom. The reason for some of the restrictive regulations is there are still doubts in the minds of some of the key public health groups, including the World Health Organization, as to the safety and efficacy of electronic cigarettes.

In the United Kingdom, the Royal College of Physicians and Public Health England have stated they believe ecigarettes to be at least $95 \%$ less risky than traditional cigarettes, but more research is needed to confirm this. Where that has led, in Europe, is to a set of regulations that require companies selling e-liquids and devices to supply information on them, including data on emissions and toxicity. So that is an obvious place where in vitro research will go.

In the European Union (EU), there really is an adverse reaction by both regulators and the population at large in certain countries to in vivo research, so we are not required to, and actually not allowed to, conduct in vivo research on tobacco products. So it is bringing forward the opportunity for in vitro research.

In the context of the U.S. FDA, the FDA regulations ask for a considerable amount of information on products, and I would assume that may also include the FDA asking for in vitro data for some products before they are allowed to be released on the market.

Dr. Wilcox: Related to the FDA perspective, it includes some of the more onerous regulations on a worldwide basis. The good part about that is it is going to drive a lot of research and requires stakeholders to work together to find out how to meet the regulatory requirements with in vitro methods.

Dr. Hartung: I think there is a good reason why it took us 50 years to show, without doubt, that smoking produces lung cancer. The reason is that the animal models are not good enough for this. It is quite difficult to produce lung cancer by smoking in rodents, so why should in vivo methods be any better for regulating even more harmless products?

So for this reason, we first need to produce safety data. It means automatically that we also need more appropriate tools that are more sensitive and that can be interpreted more unambiguously.

\section{Dr. Fowle: Good point. Rodger, would you like to add any- thing?}

Dr. Curren: Just one point on the idea of global regulations; I would hope that we are able to reach some consistency with the type of requirements that are going to be required globally. Otherwise, we can have a lot of money, time, and effort wasted in developing specific types of assays for one region versus another region.

So having as much communication and collaboration as can exist across the regulatory agencies globally would help make the science much more efficient.

Dr. Proctor: That is a really good point. There is a common theme among regulators around the world about having some doubts about the risk profile of e-cigarettes and e-liquids. Regulations are beginning to emerge related to the need to ensure that the toxicity of e-cigarette vapor is limited. So even though there is not necessarily a common approach to what tests to be used, there is, I think, a common theme of a need that appears across regulators.

Dr. Fowle: Thank you. On to the next question: What lessons can be learned from other industries about how in vitro, in silico, and/or other alternative approaches can be used to solve critical science questions?

Dr. Curren: Thank you, Jack. A good example is from a somewhat similar situation in which one area of U.S. industry, the household cleaning products industry, started advertising their products as being antimicrobial or antibacterial. By making these claims, the products came under Environmental Protection Agency (EPA) pesticide regulations, because the products were designed to kill organisms. Thus, the companies needed to register these products with the EPA, which had not been required when they were not specifically labeled as antimicrobial.

The EPA regulations at this time required that animal tests, specifically the Draize eye test, be conducted to determine potential eye irritation from these products.

Now most of these companies had not been doing animal eye irritation tests for many years (and had safely marketed the products), but when each company went individually to the agency with in vitro data for this end point, they were generally met with resistance.

What occurred then was that a small consortium was formed with a group of seven or eight manufacturers of the products with the goal of working together directly with the EPA on this situation to try and come up with a consistent in vitro testing strategy for these products.

The short conclusion here is that by working together and providing a larger pool of data, the EPA accepted this nonanimal testing strategy, and it is now an approved EPA policy. This has become a success story in utilizing in vitro methods for a regulatory purpose.

There are learnings from this that might be applicable to the situation we are discussing today. I think there were two main drivers of success. One is that companies came together in a collaborative way. This not only provided more data and a wider range of products for the EPA to look at but it also showed that no company was going to get some type of technical advantage by having a better safety test than another.

That is a good thing to remember, which in almost all industries, safety tests are not looked upon as something that should be proprietary, but should be available to everyone within the industry so that all are working on the same playing field. 
The second learning from this is that the agency itself was involved right from the start, so EPA scientists saw the data and the hazard assessment strategy as it was being developed. Thus, they were able to understand and comment on the process during its development to make it more useful for agency use.

This may be more difficult in the current situation with the Center for Tobacco Products (CTP) here in the United States, but I think we should encourage collaboration and transparency as much as possible, because that is always the best way forward.

Dr. Proctor: That is actually a really good example. In the case of e-cigarettes, there are a huge number of very small manufacturers that really do not have any research facilities or ability to do this. So it is going to need a group of people under the guidance of regulators, such as FDA, the EU, and other countries, to really pull together a common set of testing approaches for these products.

And without that kind of consortium, which includes smaller companies, then it really will become an unfair playing field. We have started to see that happen in some of the product standards developments happening, both in the United Kingdom and in the EU.

Dr. Hartung: On the one hand, it is great that in vitro toxicology has flourished because of other industries being under pressure or having the willingness to develop it. But we should also be clear that somehow e-cigarettes are unique, in that we do not have really a risk-benefit analysis as the basis of regulation, but we want to exclude risks.

Currently, we will not be able to exclude the risk of ecigarettes. This is not a safe product. It is only so much better than the terrible product it is replacing. It has been calculated by the WHO that 1 billion people will be killed because of smoking in the 21st century. Five percent of that number is still 50 million people. And no other product would be allowed to get to the market that is going to kill 50 million people.

So it is really important that we come up with a completely different risk assessment paradigm; we cannot subject ecigarettes to testing where we want to establish no risk, because that is only killing the product.

Dr. Proctor: I totally agree with Thomas there. And there is a little danger that because the comparator product, the cigarette, is so dangerous that people say, "Well, it must be safer," and, therefore, does not need testing.

So vapor products will probably have some residual risk. The health risks should be much less than those of cigarettes, but putting a strategy of testing in place will be really important.

Dr. Fowle: What innovations and test methods are required to meet emerging decision support needs? Novel assays, puffing delivery systems? How can toxicity testing continually evolve to meet the rapid evolution of new products to best inform decisions at FDA and elsewhere?

Dr. Wilcox: So the Tobacco Control Act, as enacted in 2009, gave FDA jurisdiction to regulate tobacco products. This was amended in 2016 with the deeming regulations. And it resulted in a significant departure from the way in which FDA regulates products, and it is kind of what Thomas was alluding to, and that is the FDA's position that no tobacco product is safe.

The FDA standard for drugs and devices is that of: Does it meet the standard of being safe and efficacious? The CTP now enforces a different standard as required by the Act: that is a Public Health Standard. So what does that mean? Well, it depends on how you look at the way in which the CTP is going to accept tobacco products versus drugs and devices and what innovative test methods will be required to meet this new standard.

If we use the example of FDA's premarket tobacco product application for ENDS (electronic nicotine delivery systems), we have a fairly clear idea of what information FDA is looking for. And this gets into the microsituation I alluded to earlier, and that is: What are the exact criteria of risks that FDA is looking at with electronic cigarettes?

Not unlike drugs and devices, the FDA is requiring the sponsor of new tobacco products to adequately characterize what they traditionally refer to as the intended use and exposure characteristics.

Examples of what FDA requires are as follows. They want to know what is the portal of entry? With ENDS it is inhalation. What are the absorption characteristics? Where in the respiratory tract are the substances absorbed? What substances are being inhaled? In other words, what are the ingredients?

What are the chemical and physical analyses of the ingredients, as well as the quantitative characteristics of the aerosol emissions? What is the user topography? In other words, puff frequency, duration, and intensity? What is the computation modeling to establish toxicity?

These examples suggest to me that the public health standard is not much of a departure from substantiating safety as FDA requires with drugs and devices. Clearly, the sponsor of a new tobacco product needs to meet the FDA's criteria and meet with FDA in a premarket application meeting and discuss what is required.

In the ENDS guidance document, the FDA made it very clear that in vitro methods are preferred. And I find that encouraging in their guidance document. They request that the sponsor meet with FDA in advance of an application to discuss not only the in vitro methods to be used but also if there are in vivo methods, what are they? They are encouraged to work with FDA. I found that to be promising.

Dr. Proctor: I think one of the key elements of e-cigarettes that requires us to really think about fast and high throughput screening in the toxicological testing is the fact that you have actually got probably millions of combinations of possibilities.

If you add the number of liquids available to the number of devices available and assume that any liquid can be used in any device, you have a really complex marketplace that sets a real challenge for toxicological evaluation.

Dr. Hartung: And inhalation toxicology is one of the least developed areas of toxicology, not that it is less relevant, but it is simply so costly to do, and all of the small animal research here has a lot of limits because the respiratory tract and the behavior of the animals when exposed to 
these types of things are so different that it is very difficult to carry out these studies, which come at enormous costs.

So this should be a stimulus to the development of in vitro inhalation toxicology type of approaches because they are desperately needed, and this product shows it.

Dr. Curren: Just to follow up a bit from what Thomas has said, even as we are looking at the in vitro model systems, there are so many different areas where we can try to mimic the inhalation characteristics of a human smoker. I think we can get tremendously complicated here, and we may need to do that for some of the initial studies.

But in the long run, we may try and seek somewhat of a reductionist level of activity, or type of model, so that this huge matrix of combinations of e-cigarette liquids and devices that Chris was talking about could actually be measured in a reasonable time period.

For example, do we really need tubes that represent the bronchial system or are currently available three-dimensional cultures sufficient? Just one of the many questions that needs to be answered.

\section{Dr. Fowle: Thank you; that was a very good discussion. Let us move to the next question. What role will in vitro and/or in silico approaches play in bridging data sets from a refer- ence to a similar product?}

Dr. Hartung: What is our point of reference? Traditional cigarettes are from my point of view no reference. We have the saying in our textbooks and medicine that nicotine is addictive and tar kills, but the tar is not present in ecigarettes. We are worried here about substances, which are either not in cigarettes at all, the different flavors, and stabilizers, or are substances, which we have not identified previously as a problem.

We can also not compare the new products to not smoking, to no exposure, because that is not giving us any quantification and basis for comparison. So what we desperately need is the standard e-cigarette, like we have standard cigarettes. You can buy a standardized smoke solution. It is necessary that we agree on one product to compare with, to establish at least the relative risk of different types of devices and different types of e-liquids.

Dr. Wilcox: I think developing a standardized e-cigarette is important, but just like from an FDA perspective, the FDA has not shown any interest in accepting the notion that a cigarette is a cigarette, so to speak. So when it comes to an e-cigarette application, I think they are going to want data on that particular e-cigarette.

Dr. Proctor: I think that seems to be right at the moment, that the FDA is facing an enormous challenge of the number of applications it are going to have with the existing products in the marketplace. It is going to have to find a way in which it can look at creating some foundation data sets on some products and then finding ways to bridge across those with all the other combinations. And that requires some foundation data sets to get out there, which include a set of in vitro models, as well as the chemistry and clinical data.

But then once you have that, you try and take a product that has been extensively tested and make it into a refer- ence product that can be put in to further tests among the comparators.

Dr. Hartung: The in vitro and in silico methods, first, can serve as a screen to look at what smells like a problem. Facing the large number of substances and combinations, they can help us to invest on the most risky substances to learn about them.

Dr. Curren: Certainly, along with identifying a reference e-cigarette there has to be an agreement on what specific markers of disease should be investigated with those ecigarettes. One can envision hundreds of end points that could be measured on a single reference e-cigarette, but we need to do it at the same time and thus need a smaller number of really usable markers that indicate disease.

Dr. Wilcox: I think Rodger's point is particularly important, and that is we have to work with the regulators and the scientists in the private sector as well to identify what needs to be measured from the e-cigarette to establish the potential for disease. This is very important.

\section{Dr. Fowle: Thomas, did you see any role for something analogous to "read-across" for comparing these different e-cigarettes or devices, or is that not applicable?}

Dr. Hartung: Well, "read-across" depends on the availability of data sets. Inhalation toxicology is an area that is very data poor, so we should not expect to find good coverage of these things, and we are also dealing in many of these flavors with complex mixtures. Again, not all of them are synthetic and actually defined. But still it will be one of the important tools to help us to complement possibly in vitro data sets to fill data gaps and take conclusions of what to look at or what not to look at.

The robustness and velocity of "read-across" type of approaches, I think, are lending themselves for these types of early assessments.

\section{Dr. Fowle: Thank you. Let us move to the next question. Should-and how can-industry and product manufactur- ers play a role in contributing to the e-cigarette science base?}

Dr. Proctor: I think there is an awful lot that we as manufacturers need to do. And that starts with characterizing the products that we manufacture ourselves. It is something we have been doing and capturing in a series of publications coming out this year. This research details what is present in e-cigarette vapor in terms of toxicant emissions from a simple chemistry point of view. We are starting to do a lot of work using untargeted analytical chemistry to see whether there are chemicals in vapor that you do not find in cigarettes, and if so whether these might pose risks.

And then we need to look at the consumer side of this. So what actually is the pattern of consumer use? How many ecigarette puffs do people take? What is the volume of those puffs? What is the inhalation pattern related to those puffs?

We are currently creating a large foundational data set on a couple of different e-cigarette formats, which include behavioral research, chemical analysis, a range of in vitro testing, 
systems biology approaches, and clinical research. These data will appear in a large set of peer-review publications, some that have already been published recently and more should be accepted as we go through this year.

Dr. Hartung: I think one of the important aspects is that we have barriers between the research taking place in the tobacco industry and the research done in academia and other areas. This is largely a historical piece.

The industry has a terrible reputation. It is, for a researcher, problematic. It is difficult to engage in active research with the tobacco industry, because it is instantly suspicious that you are helping them to clean a terrible product to get it to market.

And I think it is extremely important that we break down this barrier and that we find ways where the vested interest of industry is somehow disassociated from the research, which is not necessarily done in its interest.

A lot of this is taking place now with FDA using the money coming from tobacco industry and financing research into new products, but I am too often seeing that this is not addressing fast enough the challenges of the e-cigarettes and this dynamic market.

Dr. Fowle: If in vitro and/or in silico approaches are to play a key role in safety assessments and regulatory submission using bridging approaches, such as adverse outcome pathways (AOPs) through integrated approaches to testing and assessment, how do we get novel methodologies to a level of validation that would be acceptable for public health and regulatory scientists?

Dr. Wilcox: Fundamentally, this is a question that has been asked for decades now, and that is: "How do we validate alternative methods in toxicity testing?" Again, I am talking from an FDA perspective. I think this point hinges on: How does FDA handle accepting methodologies that are not necessarily validated for a particular use?

Currently, as far as I know, not only does a sponsor of a new tobacco product have the burden to substantiate the impact of a proposed product by using the public health standard in tobacco products, but the sponsor also has the burden to demonstrate that the methods he or she proposes to support his or her claim are appropriate for his or her intended use.

At the same time, it is incumbent upon the regulatory scientists to adequately understand the proposed assays and know when the data have been generated under, and meet the conditions of, well-controlled studies. It is important to understand how FDA reviews methods that are used by industry; industry research is accepted by FDA and even embraced, contrary to many other organizations outside of the tobacco industry. In other words, formal validation is important where it has application. However, on a practical basis, not all methods used by industry to support product submission will be validated.

As a result, proposed methods will be reviewed by FDA as to suitability for several factors, including whether or not they are well-controlled studies. It is especially important that we look forward to what Chris referred to as a consortium. Maybe a public/private initiative between the tobacco industry, the regulators, the research industry outside of the tobacco industry, and academia to address the specific end points, as mentioned by Rodger, that need to be measured. In addition, when methods exist that can measure these end points, looking at what methods need to be developed and possibly validated to use for tobacco products is also important to consider.

Dr. Proctor: Just recently, there has been an EU advisory report, which starts to look at the need for analysis of tobacco additives, but it goes to the same theme. There was an acceptance of wanting data that were in line with 21 st century toxicology and AOPs.

So I think regulators are starting to be more accepting of this type of data and accepting that actually it is going to take quite a long time to get some of the methodologies validated.

The other issue, at least in our laboratories, when you do the more regulatory-accepted tests, such as the Ames test and some of the cytotoxic end points, you get no response from the emissions of an e-cigarette. You obviously get a large response from cigarette smoke, but from an e-cigarette you are getting no mutagenicity and little or no cytotoxicity.

So unless we get more refined tests that may be more valid to what we are trying to look for here, we are going to be left with a blank piece of paper that just says, "There is no toxicity going on."

Dr. Hartung: In an ideal world, we would carry out clinical trial-type studies, epidemiological studies. But as long as we are looking at health effects, like the possibility of lung cancer, which develop over 15 years, these studies would need the follow-up of 20 years, and we cannot wait for this.

At the same time, we do not have reference data. We do not have animal studies. We do not have anything on these new products allowing us to form a point of reference to a formal validation study. So we will not be able to do what we have been doing for other in vitro systems by simply using bad and good guys and try to correctly capture them with the in vitro method.

So the only thing, which is left for us, is something, which is based on scientific understanding on mechanism-based evidence, and I think this is something in which the new concept of mechanistic validation is coming into play.

A lot of this will be about analyzing the quality of the evidence and the way the evidence has been integrated. For this reason, I would like to bring forward that evidence-based toxicology, which is formalizing these processes, and is also creating a distance between those who have produced the data and those who integrate them; it is actually a very interesting approach to help us sort out these data, which come necessarily from a biased background.

Dr. Curren: We have to remember that we are not working on this problem of validation of end points and models in a complete closed system-one that is only looking at tobacco and tobacco/nicotine products. There are also many other fields, and many other industries, that are looking at results from inhalation exposure to their products or their chemicals that can provide significant background information that supports or enhances what is found in the tobacco-specific research. We have to realize that that can provide enormous help.

But also a big component of this would be the fact that FDA's CTP or other international organizations are going to 
have to start commenting on what, to them, is a validation or an acceptance paradigm that can be followed, what they are looking for in terms of strength of data, what their own laboratories are producing, and is "fit for purpose" a possibility?.

Dr. Fowle: That is a good segue into our next question: What is the best method to accelerate testing approaches? Is there a place for industry, product manufacturers, tissue manufacturers, and regulatory authorities to work together?

Dr. Curren: Thomas mentioned earlier that because of historical examples of industry research exhibiting bias, it is difficult today for industry and academic researchers to collaborate. Similarly, I do not know of an arena right now where the industry scientists and regulatory scientists can actually "safely" work together. However, the ability to collaborate is a scenario that we should be pushing for, because it will make the process much more efficient, but-except for a few exceptions - this does not seem possible right now.

One example of where some success at collaboration has been achieved is a recent series of workshops that our company, the Institute for In Vitro Sciences (IIVS), has been supporting. As IIVS is a nonprofit institute, it is generally looked at as a neutral party. Therefore, we have been able to organize workshops where industry scientists, academic scientists, and regulatory scientists from FDA CTP have been able to attend.

There are the beginnings of some dialogue between regulatory researchers and industry at these meetings. It is a start and hopefully the beginning of even broader research communications. As with any interaction between two parties, if you are not face-to-face, you may have doubts about the intent of the other party. But when you start getting people together in meeting rooms and start having dinners together and start talking on a one-on-one basis, you realize that both sides may have some important things to say.

I think the continuation of programs like the workshops already described, which are well publicized, and the results of which exist in the peer-reviewed literature, will go a long way over the next few years to bring harmony to the somewhat disparate research activities of today.

Dr. Hartung: I would like to echo this. Our experience is that, in all areas across stakeholder discussions, trust is of critical importance. It is all about trust. You need to understand who is trying to sell you something and who is coming to the table for finding an honest solution. And that is something very interpersonal. That is why you need the venues like the IIVS is providing here for tobacco where the different people can start talking to each other in a protected scenario where no regulatory decision is taken from the discussions instantly.

Dr. Proctor: I totally agree with that. We have to find ways in which we can get collaboration going more efficiently and effectively. What we have been doing is trying to reach out to small groups to start those kinds of collaborations. We have been working with some of the tissue manufacturers and actually with another tobacco company, Philip Morris International, to do research studies to make sure we can validate some of the test systems.
We have also contributed to the Organisation for Economic Co-operation and Development (OECD)'s AOPs Wiki, with a couple of AOPs based on disease end points. We are trying to define key events and key relationships in these AOPs supported by data we and others are collecting on e-cigarettes.

Dr. Wilcox: I have been encouraged in the past few years by the willingness of FDA to participate in various seminars and work with, for instance, IIVS and even some of the large tobacco companies. They appear to want to go forward and work together.

The FDA would have to take the lead in that sort of effort, but I think that the interest is there. And, as made clear from the conversation here, it is that sort of collaboration that we need going forward.

Dr. Fowle: What I am hearing is that "validation" and acceptance of data from in vitro and other alternative Tox 21 methods are going to require collaboration, dialogue, and training. Success will depend on engaging industry, FDA, and other stakeholders and involve decision-makers as well as scientists to reach agreement about what information is needed to demonstrate that the new approaches are equally if not more informative and efficient than traditional approaches to inform safety decisions. This must be done in a "fit for purpose" context not only with respect to the legal and regulatory requirements but also to the specific consumer use scenario and assessment problem being addressed. Continuing interactions and dialogue is key.

In closing, I would like the panel to consider the question: What are the best ways to present the scientific information generated about how these novel in vitro/in silico approaches can be used to inform decisions in the context of regulatory, legal, economic, and other social aspects that are considered in decision-making to ensure that the new approaches are adopted and utilized in a timely manner?

Dr. Hartung: You were alluding to continuing education, and from my point of view, this is also one of the big challenges for academia. We, on one hand, have to educate the next generation of safety assessors, but also, on the other hand, we should be aware the area becomes more and more dynamic.

We will have to stop promoting the idea that when we have educated the toxicologist, he or she is then fit for life to carry out his or her job. There are also needs for developing programs to educate the regulators. Sure, they are doing this, but this could be more structured.

At the moment, we at the Center for Alternatives to Animal Testing are starting to education for free through the web on toxicology for the 21st century, evidence-based toxicology and others, and I think these are the types of things that we need more of and also more directly tailored to the needs of regulation of these types of products.

Dr. Wilcox: I think everything Thomas said is spot on. To look at it from a practical perspective, I alluded to the need to educate the regulators on what is available and the mechanistic approach to validation and so forth.

At the same time, it is incumbent upon the scientific community to ask FDA and other regulators what are the 
questions that need to be answered so that research and education can be focused on how to go forward.

We can talk about the future of cigarettes and all the different compounds that go in the cartridges and so forth, but the bottom line to get a product approved, at least in the United States, requires that some very specific and difficult questions need to be answered. We need to focus on those questions and then get the stakeholders together to work on how they can be answered.

Dr. Proctor: In the United States, there is an investment from the regulators' point of view on science. The CTP has a large number of scientists actively looking at these types of issues, and it is connected into some of the other agencies. It has an interest in learning, so it holds workshops at the CTP on specific issues, and I have spoken at one of these workshops on in vitro models.

If you look elsewhere, in Europe, for example, there is really no investment from the regulators' point of view on this work. So the European centers of research are not doing anything in this area. In that kind of scenario, I think you have to start working with regulatory science advisors, the people who are experts in the area, to start looking at the advantages that we would have if we had a suite of in vitro tests to deal with this issue.

Dr. Curren: To add on to Thomas' initial comment about education at all levels, one difficultly that we found with the EPA antimicrobial cleaning products initiative that I had talked about earlier was that many companies were not taking advantage of that policy by using the approved in vitro methods. This was because they discovered that some reviewers who were looking at the submitted in vitro data were not scientifically familiar with the types of tests being used, even though their use had become official EPA policy. There was a lack of education within the agency. Because of this and the fact that it slowed down the application process, many industry groups were not utilizing the availability of the in vitro method. Thankfully, this has now been corrected, but it is a cautionary tale.

So as we go forward-hopefully to a situation in which in vitro information becomes an important part of risk assessment for the CTP - we really need to be sure that individuals at all levels, both the scientists from industry and the scientists who are on the regulatory front lines of the agency, understand and are clear about the advantages of the in vitro tests and the situations in which they can be beneficially applied.

\section{Author Disclosure Statement}

R.D.C. is employed by a nonprofit 501(C)(3) institute that has received some contributions from the tobacco industry. He asserts he has no personal financial conflicts of interest. T.H. is consulting Underwriters Laboratories (UL) on computational toxicology, including read-across and benefits from their respective sales. C.P. is employed by British American Tobacco, a company that manufactures tobacco and nicotine products. N.W. has a consulting contract with ITG Brands. J.F. is an independent consultant who serves on a science advisory board for a company in the tobacco industry.

Address correspondence to: Dr. John "Jack" R. Fowle, III Science to Inform, LLC 155 Terrells Mountain Pittsboro, NC 27312

E-mail: jackfowle@aol.com 